\title{
An End-user Application Programme for Surveying and Computer Aided Drafting (SCAD 2006)
}

\author{
K. P. Udagepola ${ }^{1}$, A.W. Wijeratne ${ }^{1}$, Xiang $\mathrm{Li}^{2}$, Xiaozong Yang ${ }^{1}$ \\ ${ }^{1}$ Harbin Institute of Technology, China \\ ${ }^{2}$ Harbin Engineering University, China.
}

\begin{abstract}
Due to increasing use of land surveying practices along with technology advancements, a necessity for appropriate tools for task accomplishment has been occurred. A computer application program has been developed to make land surveying applications into a single user-friendly desktop operation to reduce the complexity of the surveying work. In the developed software (SCAD2006) the processing in outline and by the nature of snapshot provides a static view of activities. SCAD2006 acts as a bridge between raw data and digital maps because it provides required tools for digitalization. It benefits professional land surveyors in developing countries for efficient completion of mapping tasks. The pilot study revealed that $52 \%$ of Sri Lankan land surveyors are currently using this software, which indicates the practicability of it within a short period of time.
\end{abstract}

\section{Introduction}

With the constantly changing technology in the field of surveying and mapping, surveyors must be up-to-date to cope up with ever increasing demands for challenging tasks. The introduction of calculators with trigonometric and other scientific functions meant tremendous increases in the speed of work in the office and in the field to obtain results. Improvements in the accuracy of calculations have also been considered due to reduction of blunders and for more accurate determination of trigonometric values. Computer aided drafting has improved the speed of completion of office works of professionals in the field of geomatics. Not only the required lines drawn, but also the lettering by the amazing combination of the computer and the plotter has made the task more convenient. Even the computation of the lines themselves, whether to determine subdivision lots or rights of way could now be computed effortlessly [6].

SCAD2006 interactively explores the end-user data that are stored in digital format and a common format used by surveyors for further processing. Through the use of the "data discovery wizard" of SCAD206, the restructuring of datasets is minimized, saving time and reducing the risks associated with vulnerable changes.

The paper is organized as follows. The second section focuses on the background of field of surveying. Field calculations that are applied in the SCAD2006 will be described in Section 3. Features and capabilities of SCAD2006 will be illustrated in section 4. In section 5, advantages of SCAD2006 for end-users will be illustrated. The section 6 describes case study in Sri Lankan surveying field. The last section details specific advantages of SCAD2006 and presents general conclusions for the paper. 


\section{Background}

Surveying is a science that dates back to ancient Rome and beyond was an essential tool at the dawn of human civilization. It has also been a vital element in the development of human environment for many centuries that its importance is often neglected. It is an imperative requirement in the planning and execution of nearly every form of construction. As soon as the ancient man developed the sense of property, he invented methods for demarcating its boundaries. Land surveying is a means of making relatively large-scale, accurate measurements of the earth surfaces. Hence, the earliest surveys were performed only for the purpose of recording the boundaries of plots of a land. However, due to increasing demands of human development, surveying has been developed into a complex science [2].

There are significant scientific discoveries which could improve the practical surveying methods but still they are marginally used in current practices due to high cost involvement. The objective of this effort is to develop an efficient userfriendly and cost effective program, which utilizes the modern techniques to meet the requirements of professional land surveyors.

The modern applications of surveying are used in fields such as transportation, building construction, apportionment of land, and the field of communication. It includes the determination of the measured data, the reduction and interpretation of data to usable form and conversely, the establishment of relative position and size according to given required measurements. Thus, surveying has two similar but opposite functions: (i) The determination of existing relative horizontal and vertical position, which is used for the process of mapping, and (ii) The establishment of marks to control construction or to indicate land boundaries. It deals with geometric measurements on or beneath the earth-surface, computations of relative positions, extents etc., and the representation of these data in graphical form such as plans or maps.

Except for minor details of technique and the use of one or two minor hand-held instruments, surveying is much the same throughout the world.

A surveyor is not only responsible for providing results derived by his measurements, but also giving an indication of its quality and reliability. Whether maps or plans have to be prepared, boundaries defined and marked, designed structures layout and staked or deformations monitored, it is the surveyor who is called upon to come up with results that meet given specifications.

\subsection{Surveying instruments}

Basic surveying instrument of unknown origin is going back to the $16^{\text {th }}$ century [2]. English mathematician called Leonard Digges; had used it to measure horizontal and vertical angles. In its modern form, it consists of a telescope mounted on it to swivel both horizontally and vertically. Leveling is accomplished with the aid of a spirit level; crosshairs in the telescope which permits accurate alignment with the object sighted. After the telescope is adjusted precisely, the two accompanying scales; vertical and horizontal, will be read [4]. 


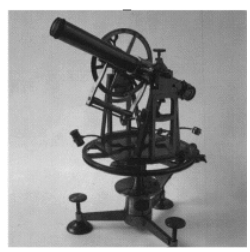

Figure1. Instrument of Theodolite Mounted on a tripod with adjustable legs

The theodolite is used in the field to obtain precise angular measurements for triangulation in road building, tunnel alignment, and other civil-engineering works. The transit is a variety of theodolite which has a telescope mounted on it which can be completely reversed, or transited.

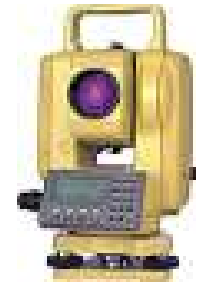

Figure 2. Instrument of Electronic Distance Measurement

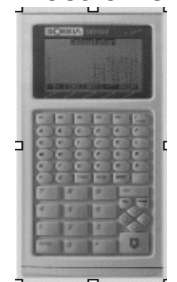

Figure 3. Data Recorder

Electronic Distance Measurement (EDM) instrument has greatly simplified an alternative technique, called traversing, for establishing a framework. In traversing, the surveyor measures a succession of distances and angles between them, usually along a traveled route or a stream. Before EDM was available, traversing was used only in flat or forest areas where triangulation was impossible. Measuring all the distances by a tape or a chain is tedious and slow, particularly if great accuracy is required, and no check is obtainable until the traverse closed, either on itself or between two points already fixed by triangulation or by astronomical observations [7]

In both triangulation and traversing, the slope of each measured line must be calculated in such a way that the map can be reduced to the horizontal and referred to sea level. A measuring tape is stretched along the ground or suspended between two tripods; in precise work corrections must be applied for the sag, for tension, and for temperature if these differ from the values at which the tape has been standardized. In work of the highest order, known as geodetic, the errors must be kept to one millimeter in a kilometer, that is, one part in $1,000,000$. 
During the past few decades, there has been a growing interest in the automation of mapping processes, and considerable progress been made in this area. Achievements in the fields of electronics, high-speed digital computers, and related technologies provided a favorable period for such progress [10].

The aspect of automation is developing rapidly, which also concerns graphic data acquisition, storage, and retrieval. Data banks are being accumulated by specialized users of topographic information, often to produce thematic maps showing soil types, vegetation classifications, and a variety of other information. Such data banks are usually organized in two parts: one for line work, such as boundaries, and the other for descriptive information or classifications. Assuming that the necessary inputs have been made to the data bank, special plats can be generated speedily. Examples of such graphics include profiles showing elevations along a selected radio propagation path and cross sections for earthwork on roads and other construction [1].

The delineation of any property in map formed by constructing lines of equal values of that property from available data points. A topographic map, for example, reveals the relief of an area by means of contour lines that represent elevation values; each such line passes through points of the same elevation. The method is not totally objective because two investigators produce different maps whenever interpolation between data points is essential for construction of the contours. In addition to topography, there are scores of geophysical, geochemical, meteorological, sociological, and other variables that are mapped routinely by the method. The availability of plotting devices in recent years has permitted mapping by computer, which reduces the effect of human bias on the final product [9].

\subsection{Field works}

Surveying measurements are of four types

(1) Horizontal lengths or distances,

(2) Vertical lengths, or differences in height or elevation,

(3) Horizontal angles measured in horizontal planes, and

(4) Vertical angles measured in vertical planes.

The data directly obtained from the field are called raw field data. They are collected by applying various methods to calculate coordinate (North, East, Elevation). 


\subsubsection{Different of methods of surveying}

Table 1 Summary of surveying field tasks

\begin{tabular}{|c|c|}
\hline Task & Description \\
\hline Traverse & $\begin{array}{l}\text { Measurement of angles and } \\
\text { distances at survey stations } \\
\text { along a predetermined route }\end{array}$ \\
\hline $\begin{array}{l}\text { Traverse and } \\
\text { offsets }\end{array}$ & $\begin{array}{l}\text { In addition to traversing, } \\
\text { measurement of perpendicular } \\
\text { distance along the survey lines } \\
\text { to features that needed to be } \\
\text { shown on a map. }\end{array}$ \\
\hline $\begin{array}{l}\text { Traverse and } \\
\text { radiation }\end{array}$ & $\begin{array}{l}\text { Measurement of angles and } \\
\text { distances from traverse } \\
\text { stations to necessary features. }\end{array}$ \\
\hline Intersection & $\begin{array}{l}\text { Establishing a ground position } \\
\text { from two or more known } \\
\text { positions with angles, } \\
\text { distances or both. }\end{array}$ \\
\hline Resection & $\begin{array}{l}\text { Establishing the position of an } \\
\text { occupied ground point from } \\
\text { angle measurements to two or } \\
\text { more known points. }\end{array}$ \\
\hline $\begin{array}{l}\text { Resection and } \\
\text { radiation }\end{array}$ & $\begin{array}{l}\text { Measurement of angles and } \\
\text { distances from resected } \\
\text { station to necessary ground } \\
\text { features. }\end{array}$ \\
\hline Setting out & $\begin{array}{l}\text { Laying out on ground, } \\
\text { measurements compiled from } \\
\text { a plan. }\end{array}$ \\
\hline Levels & $\begin{array}{l}\text { Measuring relative elevations } \\
\text { between ground or structural } \\
\text { points. }\end{array}$ \\
\hline
\end{tabular}

\subsubsection{Types of raw data.}

(i) Traverse data (Length, Horizontal bearing and Vertical bearing)

(ii) Offset (Length and offset length)

(iii) Level Line data (Back sight, Intermediate sight and Forward sight)

(iv) Unfiltered Coordinates (Point No, North, East, Elevation, code)

(v) Three point problem data (Three known point coordinates, two angles from the reference point)

(vi) Two point problem data (Two known point coordinates, six angles)

(vii) Radiation (Length and bearing)

(viii) Resection

(a) Two known coordinates and two bearing 
(b) Two known coordinates two length and side

(c) Two known coordinates, a length and a bearing

Above described length can get unit of meter or chains and bearing Degree, Minutes Seconds or grads.

The system needs to

- Feed or download data

- Process fed data

- Display processed data in tabulated or graphical form

- Computation of various details necessary for the final product

- Production of the final result with attributes.

- Storage of all fed in, computed and output data in files

In storing data, it is a great advantage when the file extension provides an indication of what type of data is stored in the file ( ${ }^{*}$.dat- Coordinate, * .trdTraverse raw data, *.Ird- Level raw data, *.ext- area of land parcel details). Each file extension has a specific meaning. In any time, it is possible to detect the type of data available in it.

The system requires to convert the data gathered by any of the above methods into the final plan should necessarily be fast and accurate. The software should also be compatible with the popular CAD environment because a client should be able to process the final result in his personal computer.

\subsection{Field surveyors requirements}

The required system (figure 4) is accomplished to saves time significantly in general operations.

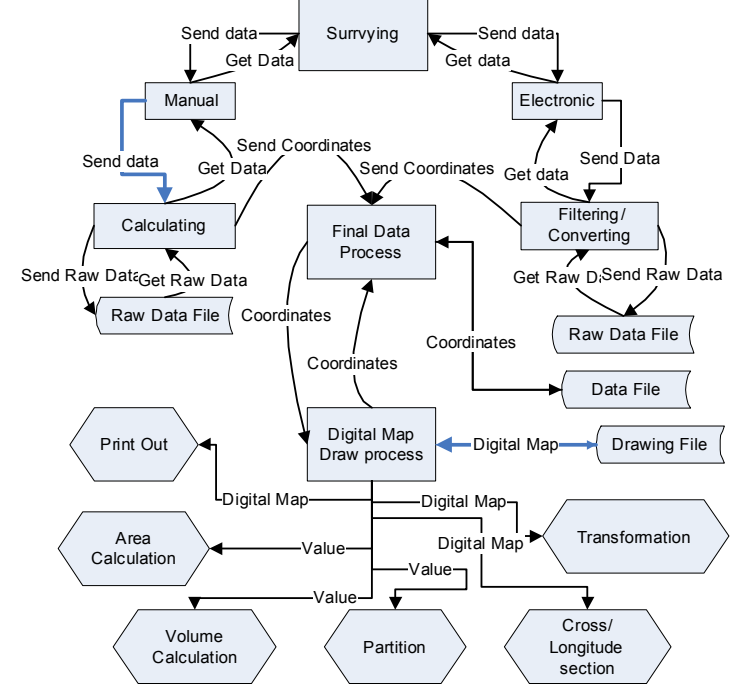

Figure 4. Overview of the system

It needs to provides quick extract of known and unknown coordinate points from drawing, fast extract grid coordinates from drawing (Engineering Surveys) and write spot heights in proper way, fast data format conversion (Field raw data to

53 K. P. Udagepola, A.W. Wijeratne, Xiang Li, Xiaozong Yang 
coordinate file or coordinate format to field raw data format), fast calculation of coordinates for offset method and simultaneously draws calculated boundaries, quick building up of script file and draw boundaries for contour maps, fast drawing of longitude section plan (with text) and able to generate data file from Longitude Section and cross section plan (with text) and able to generate data file from Cross Section and fast level line calculation.

\section{Field surveying calculation}

The program SCAD2006 has been developed giving much emphasis on the practical adaptability by covering all the aspects of surveying to generate accurate digital map. Following details the algorithms used in the software.

\subsection{Traverse calculating}

Any point on the north coordinate

$N_{i}=N_{(i-1)}+L_{i} * \operatorname{Cos} \theta_{i}$

Any point on the east coordinate

$E_{i}=E_{(i-1)}+L_{i} * \operatorname{Sin} \theta_{i}$

Where

$i=1 \ldots n$

$N_{0}=$ Start point north coordinate

$N_{i}=$ North coordinate at station "i"

$E_{0}=$ Start point east coordinate

$E_{i}=$ East coordinate at station " $i$ "

$L_{i}=$ Length of traverse line between station " $i$ " \& " $i+1$ "

$\theta_{i}=$ Bearing of traverse line between station " $i$ " \& " $i+1$ "

\subsection{Adjustment of partial co-ordinates}

When a traverse is run between two fixed stations, the totals of the partial coordinates (with regard to the sign) should be equal to the differences between the co-ordinates of the fixed stations. Similarly, when a traverse is run as a closed loop, which commences and closes on the same station, the partial coordinates should sum to zero.

Neither of these conditions is fulfilled in practice, due to inevitable errors, and the closing errors in the partial easting and partial northing sums must be distributed round the traverse.

The traverse will be closed when the obtained error is less than the allowed error and will be distributed to traverse lines. A Varity of methods are available for partial co-ordinate adjustment, the two most commonly used methods are;

(1) Bowditch's Method

(2) Transit Method

Both methods alter the line lengths and the adjusted bearings, but the Transit method disturbs the bearings less than the Bowditch's method. The Bowditch's method is preferred by the reason of its simplicity and all algorithms for SCAD2006 have also been based on it.

\subsection{Level line calculation}


Any foresight elevation coordinate is given by;

$Z_{i}=Z_{(i-1)}+\left(B S_{(i-1)}-F S_{i}\right)$

Any intermediate elevation coordinate is given by;

$Z_{j}=Z_{j}+\left(B S_{(i-1)}-I S_{j}\right)$

Where

$i=1 \ldots n$ and $Z_{0}=$ Start point elevation coordinate

" $"$ " is station number and " $n$ " is number of points of instrument stations.

" $\mathrm{p}$ " is intermediate station number

$Z_{i}=$ Elevation coordinate at station "i".

$B S=$ Back sight reading

$F S=$ Fore sight reading

$I S=$ intermediate sight reading

Adjustment on level line: Since there are always errors in survey, a limit has to be set for the permissible (i.e. acceptable) error in any leveling task. The error permissible depends upon the type of the task.

Offsets and running Offsets: An offset has been defined as a short measurement taken (raised) at right angles from the chain line to the point of detail to be surveyed. Offsets are said to be 'raised' and 'measured'.

\subsection{Area Calculations}

The surveyor is often required to determine areas of land and sometimes to subdivide areas and fix new boundaries.

If a closed traverse has been coordinated, or the boundary of a property or a cross-section is defined by coordinated points, then it is possible to calculate the area of the figure concerned directly from the co-ordinates without plotting the figure on a paper. Figure 6 shows a four-sided closed loop traverse. It is evident that the area of the figure $A B C D$ (figure 5 ) is equivalent to the addition and subtraction of trapeziums

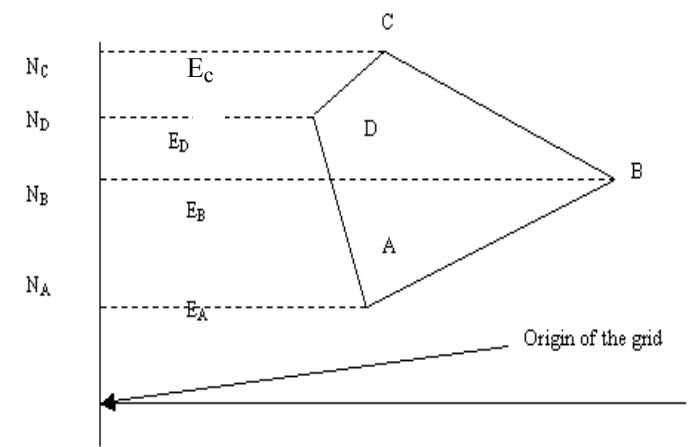

Figure 5. Boundary of Land parcel

Area $=0.5\left(N_{B}-N_{A}\right)\left(E_{A}+E_{B}\right)+0.5\left(N_{C}-N_{B}\right)\left(E_{B}+E_{C}\right)-\left(0.5\left(N_{C}-N_{D}\right)\left(E_{C}+\right.\right.$ $\left.\left.E_{D}\right)+0.5\left(N_{D}-N_{A}\right)\left(E_{D}+E_{A}\right)\right)$

$N_{A}=$ north coordinate of point number $A$

$E_{A}=$ east coordinate of point number $A$ 
$N_{B}=$ north coordinate of point number $\mathrm{B}$

$E_{B}=$ east coordinate of point number $\mathrm{B}$

$N_{C}=$ north coordinate of point number $C$

$E_{C}=$ east coordinate of point number $C$

$N_{D}=$ north coordinate of point number $\mathrm{D}$

$E_{D}=$ east coordinate of point number $\mathrm{D}$

\subsection{Partitioning}

We can separate required Land area from a given plot using two methods.

(1) Creating a parallel line for to a given line on the map which can measure four directions illustrated in figure 6.

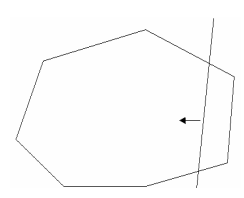

(a)

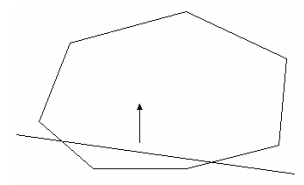

(c)

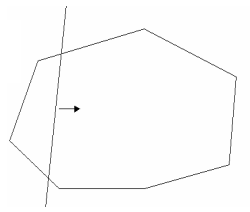

(b)

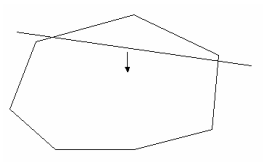

(d)

Figure 6. A Line moves land partition methods. (a) The line moves east direction, (b) the line moves west direction, (c) the line moves north direction (d) the line moves south direction.

Under this method, system creates a parallel line and moves it towards the given direction until the required area is generated.

\section{Begin process:}

$A_{\text {req }}=$ required partition area

$A_{\text {org }}=$ Original land parcel area

$$
\begin{gathered}
n=\operatorname{Fix}\left(\frac{A_{\text {org }}}{A_{\text {req }}}\right) \\
A_{\text {total }}=A_{\text {req }} \times n \\
\text { If }\left(\mathrm{A}_{\text {org }}<\mathrm{A}_{\text {req }}\right) \text { Then }
\end{gathered}
$$

Display massage box

\section{Else}

For $i=0$ To $(n-1)$

If $\left(\mathrm{A}_{\text {org }}<\mathrm{A}_{\text {req }}\right)$ Then $\mathrm{A}_{\text {total }}=\mathrm{A}_{\text {total }}-\mathrm{A}_{\text {req }} \times \mathrm{i}$

Line is moved $\delta x$ (small distance) to given direction

Calculate $A_{i}$

Do Until $\left(A_{\text {total }}>A_{i}\right)$ or $\left(A_{\text {total }}=A_{i}\right)$

Line is moved $\delta x$ (small distance) to given direction

Calculate $A_{i}$

\section{Loop}


Draw the partition line

Next $I /$ *Where; $A_{i}=$ Calculate new existing area within new land parcel* $/$

End process

(2) Fixing a point on the boundary of the map. Other point will travel along the boundary to the given direction until it generates the required area. There are two methods (Figure 7) available to rotate in the required direction.

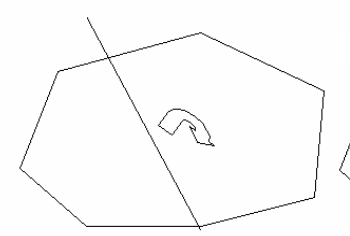

(a)

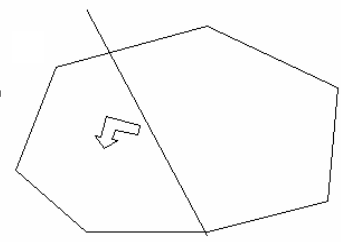

(b)

Figure 7. A Line rotates land partition methods. (a) The line rotates right hand direction, (b) the line rotates left hand direction.

Begin process:

$A_{\text {req }}=$ required partition area

$A_{\text {org }}=$ Original land parcel area

If $\left(A_{\text {org }}<A_{\text {req }}\right)$ Then

Display massage box

Else

Line is rotated $\delta x$ (small distance) given direction

Calculate $A$

Do Until $\left(A_{\text {total }}>A_{i}\right)$ or $\left(A_{\text {total }}=A_{i}\right)$

Line is rotated $\delta x$ (small distance) to given direction on the boundary

Calculate $A$

Loop

Draw the partition line

${ }^{*}$ Where: $A=$ Calculate new existing area within new land parcel on the ${ }^{*} /$

End process

\subsection{Transformation}

Most of the times, newly surveyed boundaries are superimposed to an exiting plan where the coordinates are not exactly the same. Then it is required to transform new boundaries according to exiting coordinates. But both coordinates can not be exactly matched each other, which require following algorithm. Then it will generate the output required by the surveyor.

(1) Boundary move and rotate method (MR).

(2) Boundary move, rotate and change methods (MRC). Some adjustment theories in surveying science can be used here.

Begin MR process:

$$
X_{R}=\frac{\sum_{i=1}^{n} X_{R, i}}{n}
$$




$$
\begin{gathered}
Y_{R}=\frac{\sum_{i=1}^{n} Y_{R, i}}{n} \\
X_{C}=\frac{\sum_{i=1}^{n} X_{C, i}}{n} \\
Y_{C}=\frac{\sum_{i=1}^{n} Y_{C, i}}{n}
\end{gathered}
$$

Where

$X_{R}=$ required system mean East coordinate

$Y_{R}=$ required system mean North coordinate

$X_{R, i}=$ required system $i^{\text {th }}$ point's East coordinate

$Y_{R, i}=$ required system $i$ th point's East coordinate

$X_{C}=$ new surveyed mean North coordinate

$Y_{C}=$ new surveyed mean North coordinate

$X_{C, i}=$ new surveyed system $i^{\text {th }}$ point's East coordinate

$Y_{C, i}=$ new surveyed system $i^{\text {th }}$ point's North coordinate

$i=1,2, \ldots n$

$n=$ number of selected points

$N=$ number of transformation points

$X_{2, i}=X_{2, i}-\left(X_{C}-X_{R}\right)$

$Y_{2, i}=Y_{2, i}-\left(Y_{C}-Y_{R}\right)$

$\theta_{1, i}=\tan ^{-1} \frac{Y_{C}-Y_{I, i}}{X_{C}-X_{1, i}}$

$\theta_{2, i}=\tan ^{-1} \frac{Y_{C}-Y_{2, i}}{X_{C}-X_{2, i}}$

$\delta \theta_{i}=\theta_{1, i}-\theta_{2, i}$

$\delta \theta=\operatorname{sign}\left(\delta \theta_{i}\right) \times \min \left(\delta \theta_{i}\right)$

Newvalues

$\theta_{2, j}=\delta \theta+\theta_{2, j}$

$X_{2, j}=X_{R}+\left(\sqrt{\left(X_{R}-X_{2, j}\right)^{2}+\left(Y_{R}-Y_{2, j}\right)^{2}}\right) \times \cos \theta_{2, j}$

$Y_{2, j}=Y_{R}+\left(\sqrt{\left(Y_{R}-X_{2, j}\right)^{2}+\left(Y_{R}-Y_{2, j}\right)^{2}}\right) \times \sin \theta_{2, j}$

Where

$\theta_{1, i}=$ Bearing between line of required system center to $i^{\text {th }}$ point

$\theta_{2, i}=$ Bearing between line of new system center to $i^{\text {th }}$ point

$\theta_{2, j}=$ adjusted bearing between line required system center to adjusted to $j$

point

$i=1,2 \ldots n$

$j=1,2, \ldots n, n+1, \ldots . N$

$X_{2, j}=$ required boundary $j^{\text {th }}$ point east coordinate

$X_{2, j}=$ required boundary $j$ th point north coordinate

End MR process 
Begin MRC process:

$$
\begin{gathered}
X_{R}=\frac{\sum_{i=1}^{n} X_{R, i}}{n} \quad Y_{R}=\frac{\sum_{i=1}^{n} Y_{R, i}}{n} \\
X_{C}=\frac{\sum_{i=1}^{n} X_{C, i}}{n} \quad Y_{C}=\frac{\sum_{i=1}^{n} Y_{C, i}}{n}
\end{gathered}
$$

Where

$X_{R}=$ required system mean East coordinate $Y_{R}=$ required system mean North coordinate $X_{R, i}=$ required system $i^{\text {th }}$ point's East coordinate $Y_{R, i}=$ required system $i^{\text {th }}$ point's East coordinate $X_{C}=$ new surveyed mean North coordinate $Y_{C}=$ new surveyed mean North coordinate $X_{C, i}=$ new surveyed system $i^{\text {th }}$ point's East coordinate $Y_{C, i}=$ new surveyed system $i^{\text {th }}$ point's North coordinate $n=$ number of selected points $N=$ number of transformation points

$i=1,2 \ldots . n$

$D S_{0}=0 ; D N_{0}=0 ; D X_{0}=0 ; D Y_{0}=0 ; T S Q_{0}=0$

$D S_{i}=\sum_{i=1}^{n}\left(\left(X_{1, i}-X_{R}\right) *\left(X_{2, i}-X_{C}\right)+D S_{i-1}\right)$

$D N_{i}=\sum_{i=1}^{n}\left(\left(X_{1, i}-X_{R}\right) *\left(Y_{2, i}-Y_{C}\right)+D N_{i-1}\right)$

$D X_{i}=\sum_{i=1}^{n}\left(\left(Y_{1, i}-Y_{R}\right) *\left(X_{2, i}-X_{C}\right)+D X_{i-1}\right)$

$D Y_{i}=\sum_{i=1}^{n}\left(\left(Y_{1, i}-Y_{R}\right) *\left(Y_{2, i}-Y_{C}\right)+D Y_{i-1}\right)$

$T S Q_{i}=\sum_{i=1}^{n}\left(\left(X_{1, i}-X_{R}\right)^{2}+\left(Y_{1, i}-Y_{R}\right)^{2}+T S Q_{i-1}\right)$

$\mathrm{AL}=\frac{\left(\mathrm{DY}_{\mathrm{n}}+\mathrm{DS}_{\mathrm{n}}\right)}{\mathrm{TSQ}_{\mathrm{n}}}$

$\mathrm{O}=\frac{\left(\mathrm{DX}_{\mathrm{n}} \mathrm{DN}_{\mathrm{n}}\right)}{\mathrm{TSQ}_{\mathrm{n}}}$

$\mathrm{XXB}=\mathrm{X}_{\mathrm{C}}-\left(\mathrm{O} \times \mathrm{Y}_{\mathrm{R}}\right)-\left(\mathrm{AL} \times \mathrm{X}_{\mathrm{R}}\right)$

$Y Y B=Y_{C}-\left(A L \times Y_{R}\right)+\left(O \times X_{R}\right)$

New System coordinates

$X_{2, j}=X X B+\left(A L \times X_{2, j}+O \times Y_{2, j}\right)$

$Y_{2, j}=Y Y B+\left(A L \times X_{2, j}-O \times Y_{2, j}\right)$

Where

59 K. P. Udagepola, A.W. Wijeratne, Xiang Li, Xiaozong Yang 
$j=1,2, \ldots n, n+1, \ldots . . N$

$X_{2, j}=$ required boundary $j^{\text {th }}$ point east coordinate

$X_{2, j=}$ required boundary $j^{\text {th }}$ point north coordinate

End MRC process

\section{The software}

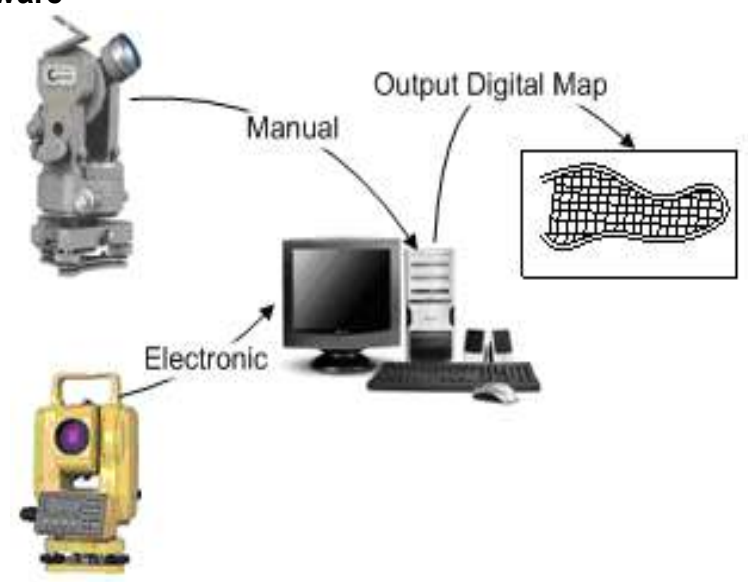

Figure 8. Required system

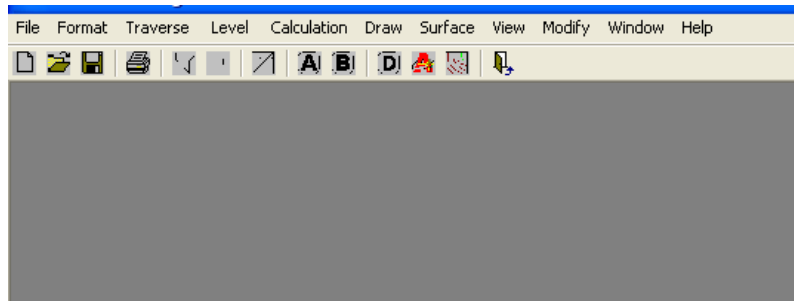

Figure 9. Main menu of SCAD2006

SCAD2006 (figure 9) is developed in MS Visual Basic as "a professional surveyor", with all the tools for creating and maintaining a surveying and computer aided drafting (figure 8), and an interface designed for outdoor use with a PC and a tablet PC.

The principal functions of SCAD2006 are;

- Draw points, arcs, polygons, annotations. While drawing, various help functions are available (Snap Node/Near, Lock Angle, Close arc about the initial node, Insert/modify the coordinates via the keyboard, etc.).

- Manage alphanumeric information in the database linked to graphic objects, with the possibility of editing, adding or eliminating any attribute, of selecting objects, executing queries, visualizing graphic elements on the basis of the associated attribute.

- Georeference vector and raster data, based on both control points and the warping of non-georeferenced data onto georeferenced ones.

- Convert coordinates among the most widely used projection systems of the world.

- Carry out main topological operations such as superimposing themes (union, intersection and identity), union of adjacent features based on associated attributes (dissolve arcs and polygons), clipping, buffer creation about points, 
lines and polygons, building topology, interactive correction of topological errors, etc.

- Manage raster data. It is possible to accomplish raster clipping and mosaics and to make transparent any defined colour.

- Print your projects by scale or entire page, personalizing the print layout to your liking.

- Directly acquire points and arcs from the GPS or perform tracking operations.

- Create personalized forms to guide input of data while surveying.

- Gather information with extreme flexibility and freedom thanks to Easy Note.

The proprietary functions above mentioned make SCAD2006 a complete, unique and valuable tool for those who wish to directly apply the technology of surveying and mapping field on-site.

\section{Double level for users}

Prior to beginning a mapping excursion, it is necessary to properly prepare the project to be surveyed. SCAD2006 provides the possibility to build a project at two levels.

The first level is; the manager that sets all the characteristics of the project for which the data collector will use in the field. The second level concerns the surveyor who physically carries out the mapping. The surveyor may be the manager or a different person. The surveyor will work within the simplified bounds of the interface prepared by the manager for the quick insertion of information.

The possibility of a double-tiered use can further impose greater uniformity among different surveyors. The same legends, symbols, observation tables and data gathering may be imposed for all surveyors. In this way, also the difference between corporate and individual information gathered in the field can be reduced [3] [5] while working, for example, in an industrial, national or regional mapping project.

\section{Case Study of SCAD2006 users in Sri Lanka}

Sri Lanka is an island where per capita land resources are shrinking rapidly due to increasing population. The demand for land for agriculture, housing, and industry is ever increasing over the past few decades. Therefore, an accurate and up-to-date plans and maps have become indispensable for proper management of this dwindling resource.

The country or the Sri Lankan surveyor does not have sufficient financial resources for sweeping changes in surveying techniques. Software that speeds up the surveyor's computations and plan production will help them immensely for effective, efficient and error free output. This would also encourage more surveyors to adopt computer-based techniques for their work.

Figure 10 shows the composition of surveyors those who have been using electronic surveying instruments and computer aided mapping for their task completions through years 2000 to 2006 . According to the results there are only about $30 \%$ of the surveyors those who have used electronic surveying instruments in 2000 while it has gone up by 2006 (40\%). In 2000, the percentage

61 K. P. Udagepola, A.W. Wijeratne, Xiang Li, Xiaozong Yang 
of surveyors those who have been using computer aided techniques for mapping purposes is only about $15 \%$. It is evident that, when it becomes 2006 , the number has gone up to $68 \%$. It is further disclosed that there are about $52 \%$ of the surveyors currently using SCAD2006 for their professional activities. Therefore, significant increase in the usage of both electronic surveying instruments and computer aided mapping by 2006 could be due to the familiarization with user-friendly desktop environment provided by SCAD2006. A calculator or a CAD package to produce the plan does computations of the data gathered. The final result could be a hard copy (98\%) or a soft copy $(2 \%)$.

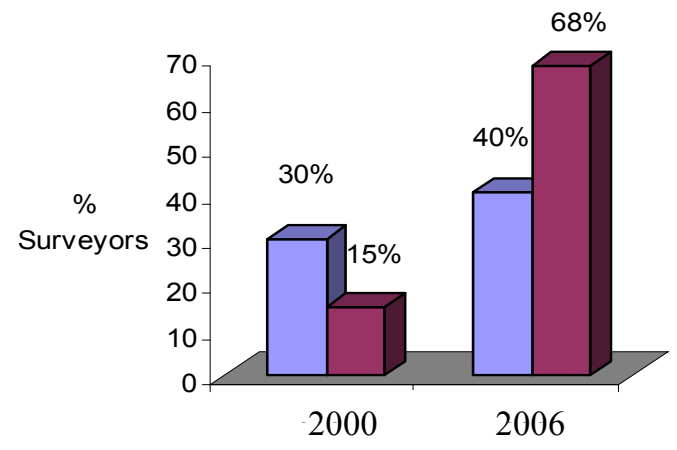

$\square$ Eectronic instruments $\square$ Computer aided mapping

Figure 10. Composition of surveyors using electronic instruments \& computer aided mapping $2000-2006$

\section{Conclusions}

With the advancement of technology in measuring techniques, giving high accuracy in positioning, have made the surveyor's task more exacting. The demand from all organizations and individuals is for fast and accurate information. Many professionals in surveying have the organizational and financial capability for fast field operations. Others pool their resources to the same end. However, their computation and plan production sector has to be quick and reliable.

The SCAD2006 is an application program that can be used to derive surveying calculations of a given set of surveyors with high precision. All mathematical calculations of land surveying are included in the calculations, and no secondary sampling system is required

In spite of technological advances, there are members of the professionals who, for various reasons continue with time tested field methods in their day-to-day operations.

In all the situations, whatever the field procedure, the software discussed here meets the requirements efficiently and accuracy of data processing and delivering the output. 
SCAD2006 has been built on the requirements of the practicing surveyor, and is being constantly improved and updated and updates are made available to users but total software size is $10 \mathrm{MB}$. It is great advantage to the users.

The popularity of SACD2006 within a short period of time revealed that the demand for such user-friendly and cost effective software is ever increasing.

\section{References}

J. Anderson, and E. Mikhail, 1998, Surveying Theory and Practice, $7^{\text {th }}$ Edition WCB/McGraw-Hill, Boston, 1998, pp. 44-52.

Britannica Ltd., 1994-2002, Encyclopedia Britannica, Inc., Britannica 2002 Deluxe Edition,.

B. Brodaric,2004, "The design of GSC FieldLog: ontology-based software for computer aided geological field mapping", Computers \& Geosciences 30(1), pp. 5-20.

J. Clendinning, and J.G. Olliver,1976, The Principal Of Surveying, $1^{\text {st }}$ ed., Educational Publications Department

M. D. Donatis, and L. Bruciatelli,2006, "MAP IT: The GIS software for field mapping with tablet pc", Computers \& Geosciences, 32 (5), pp. 673-680,.

B. Fosburgh, and J.V.R. Paiva,2001, "The changing role of the surveyor", 2001Conference sessions, American Congress on Surveying \& Mapping

H. Kahmen, and W. Faig,1988, "Surveying", Walter de Gruyter \& Co

W. Ritchie, M.Wood, R. Wright, and D. Tait,1988 "Surveying and Mapping for field scientists", Longman Group UK Ltd.

P.L. Weaver, N. Lamrou, and M. Walkley, 1988, Practical SSADM, Ver 4, $2^{\text {nd }}$ ed, Financial Times professional Ltd.

W.S. Whyte, and R. E Paul, 1985, Basic Metric Surveying, $3^{\text {rd }}$ ed. Reading, Butterworth \& Co.Publishers Ltd. 